Chimia 46(1992) 119-122

(C) Schweiz. Chemiker-Verband; ISSN 0009-4293

\title{
The Vinylogous Wolff Rearrangement Catalyzed with Rh" Complexes
}

\author{
Shahrokh Motallebi* and Paul Müller
}

Abstract. Decomposition of $\beta, \gamma$-unsaturated diazoketones has been investigated in the presence of $\mathrm{Rh}_{2}(\mathrm{OAc})_{4}$ catalyst. These reactions produce exclusively the products of the vinylogous Wolff rearrangement with higher yields than $\mathrm{Cu}^{\mathrm{Il}}$ catalysts. Chiral catalysts such as tetrakis [methyl (S)-5-oxopyrrolidine-2-carboxylate]dirhodium(II) and $\mathrm{Cu}^{\prime}$ bis(oxazoline) complexes, which are effective catalysts for the inter and/or intramolecular cyclopropanations reactions, catalyze the vinylogous Wolff rearrangement to produce the expected products with low levels of enantioselectivity.

\section{Introduction}

The thermocatalytic $\left(\mathrm{Ag}_{2} \mathrm{O}, \mathrm{MeOH}\right.$, $\Delta)$ or photochemical $(\mathrm{h} v, \mathrm{MeOH})$ decomposition of $\beta, \gamma$-unsaturated diazoketones leads not only to the expected esters derived from the normal Wolff rearrangement, but also to isomeric secondary products, which are derived from a skeletal rearrangement. In contrast, the $\mathrm{Cu}^{\mathrm{II}}$-catalyzed decomposition of these unsaturated diazoketones affords only the rearranged $\gamma, \delta$-unsaturated-acid derivatives. The process leading to the rearranged products has been termed 'vinylogous Wolff rearrangement' [1][2]. The reaction pathway for this transformation is believed to involve formation of a highly strained bicyclo[2.1.0]pentanone intermediate (Scheme 1), originating from an intramolecular carbenoid addition to the olefinic bond. The intermediate fragments to a $\beta, \gamma$-unsaturated ketene which is captured by added nucleophiles such as $\mathrm{CH}_{3} \mathrm{OH}$ to produce the stable rearrangement products.

Over the recent years, $\mathrm{Rh}^{\mathrm{II}}$-derived catalysts have been recognized to exhibit particular efficiency for reactions of diazoketones. In their presence, diazoketones decompose to metallocarbenes which participate in addition reactions to double bonds and in insertions into $\mathrm{C}-\mathrm{H}$ bonds. The Wolff rearrangement is not observed, when diazoketones are decomposed with $\mathrm{Rh}^{\mathrm{Il}}$. Recently, Doyle et al. reported that novel rhodium(II) catalysts such as tetrakis[methyl (S)-5-oxopyrrolidine-2carboxylate]dirhodium(II) are very efficient in the intramolecular cyclopropanation of allylic diazoacetates, and produce the corresponding 3-oxabicyclo[3.1.0]hexan-2-ones (see Scheme 3) with high chemical yields and very-good-to-excellent enantioselectivities [3]. The $\mathrm{Rh}^{\mathrm{II}} \mathrm{com}$ plexes are in some respect complementary to the chiral $\mathrm{Cu}^{\prime}$ bis (oxazoline) complexes of $C_{2}$ symmetry, which catalyze the intermolecular cyclopropanation of olefins with high enantioselectivity [4-6].

The structural analogy between the allylic diazoacetates and the $\beta, \gamma$ unsaturated diazoketones suggests the possibility of realizing the vinylogous Wolff rearrangement with $\mathrm{Rh}^{\mathrm{II}}$ (or $\mathrm{Cu}^{\mathrm{l}}$ ) catalysts. Chiral $\mathrm{Rh}^{\text {Il }}$ or $\mathrm{Cu}^{\mathrm{I}}$ catalysts should allow the generation of a chiral tertiary or quaternary centre, provided the diazoketone is appropriately substituted.

\section{Results and Discussion}

Some substituted $\beta, \gamma$-unsaturated diazoketones (1-5) which are known to undergo the vinylogous Wolff rearrangement with $\mathrm{Cu}^{\mathrm{II}}$, and produce rearranged esters (6-10) with a chiral centre were synthesized following the procedures reported in the literature [1][7][8]. The rearrangement reactions were performed by slow $(24 \mathrm{~h})$ addition of the $\beta, \gamma$-diazoketones in $\mathrm{CH}_{2} \mathrm{Cl}_{2}$ to a $\mathrm{CH}_{2} \mathrm{Cl}_{2}$ solution of the $\mathrm{Rh}^{\text {"l }}$ catalyst $(2.0 \mathrm{~mol}-\%)$ and a three-fold molar excess of alcohol (MeOH, benzyl alcohol). One run in which $\mathrm{CuI} \cdot \mathrm{P}(\mathrm{OMe})_{3}$ was employed as catalyst was carried out in toluene. At the end of the addition, the reaction mixture was passed through a short column of silica gel, which was washed exhaustively with $\mathrm{CH}_{2} \mathrm{Cl}_{2}$ to remove the catalyst. After evaporation of the solvent, the products

*Correspondence: Dr. S. Motallebi Département de Chimie Organique Université de Genève

30, quai Ernest-Ansermet

CH-1211 Genève 4

Scheme 1<smiles>[R]C(=O)CCC([R])=C([R])[R]</smiles><smiles>[R]C([R])=C([R])CC(N)C(=O)CC</smiles><smiles>[R]C(=C)C([R])([R])C=C=O</smiles><smiles>[R]C(=C)C([R])([R])CC([R])=O</smiles> 
Table 1. Decomposition of Diazoketones in Presence of $R h^{\prime \prime}$ and $C u^{\prime \prime}$ Catalysts

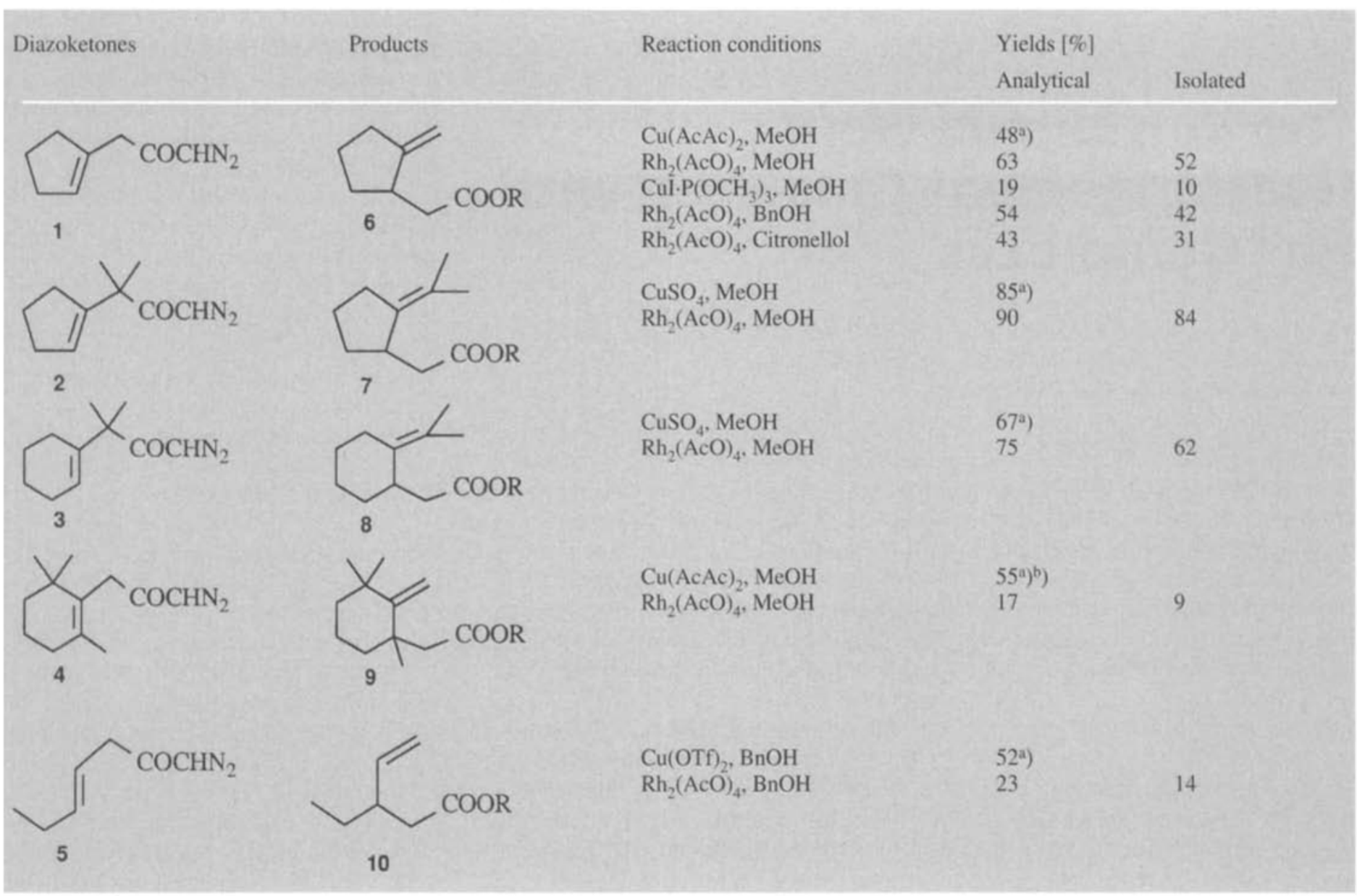

a) From [1]. ") From [8].

were separated by column chromatography and identified by comparison of their spectroscopic data given in the literature.

The results are summarized in Table 1. It is interesting to note that the $\mathrm{Rh}_{2}(\mathrm{OAc})_{4}$ catalyzed decomposition of the $\beta, \gamma$ unsaturated diazoketones leads exclusively to products of vinylogous Wolff rearrangement producing in most cases higher yields than are obtained with $\mathrm{Cu}^{\mathrm{II}}$. Use of $\mathrm{Cu}^{\mathrm{l}}$ as catalystalso results in the vinylogous Wolff rearrangement, but the chemical yield is lower than with both $\mathrm{Rh}^{\mathrm{II}}$ or $\mathrm{Cu}^{\mathrm{II}}$.

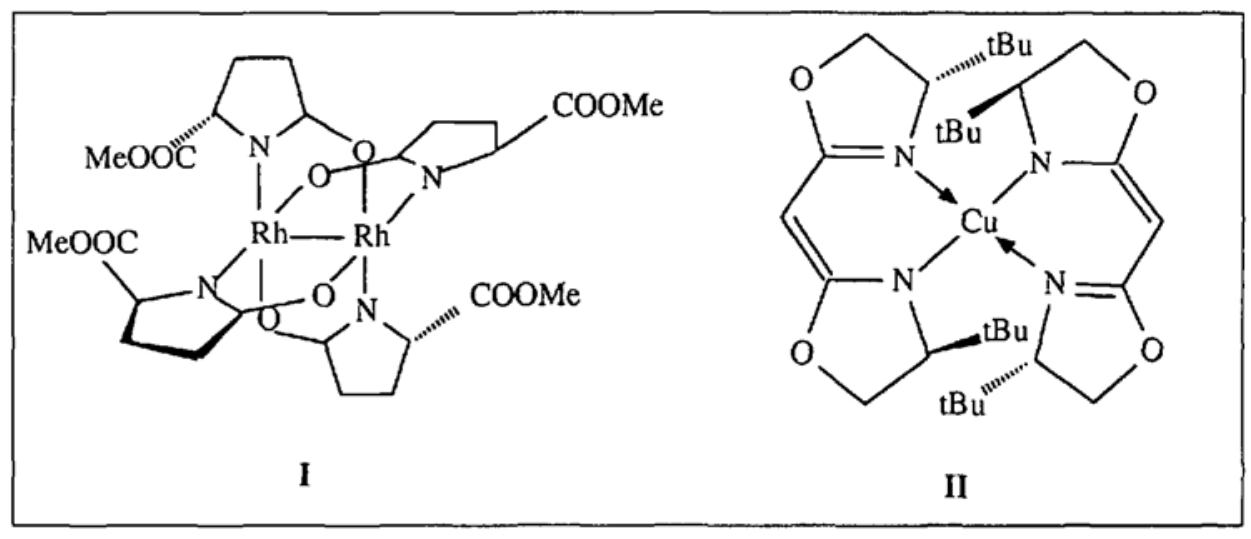

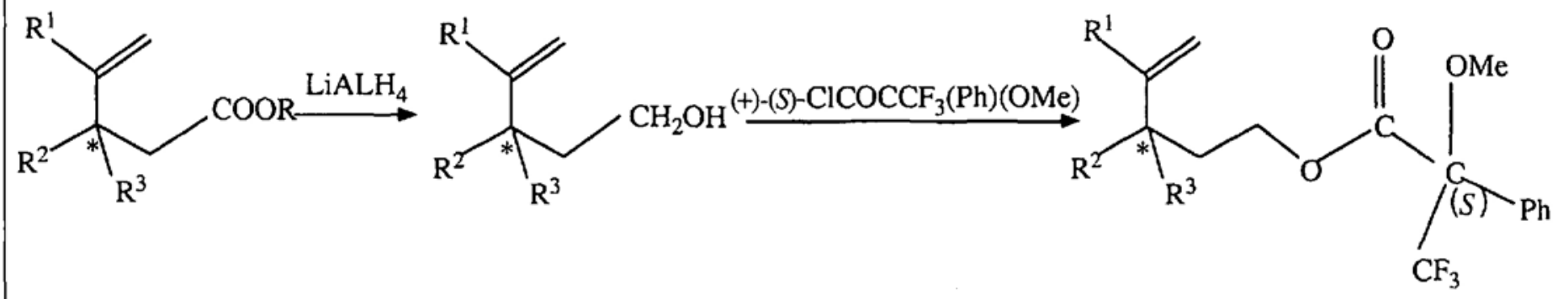


Decomposition of the same diazoketones in the presence of tetrakis[methyl (S)-5-oxopyrrolidine-2-carboxylate]dirhodium(II) $\left(\mathrm{Rh}_{2}(\mathrm{MEPY})_{4}\right)$ (I) under the reaction conditions described above, resulted in a significant reduction of the yield compared to those obtained with $\mathrm{Rh}_{2}(\mathrm{OAc})_{4}$. No improvement was achieved when the decomposition was carried out at higher temperature (refluxing $\mathrm{CH}_{2} \mathrm{Cl}_{2}$ ). We reasoned that the lower yield was due to the presence of $\mathrm{MeOH}$, which was added for interception of the ketene, since it is known that $\mathrm{Rh}_{2}(\mathrm{MEPY})_{4}$ slowly decomposes in the presence of $\mathrm{MeOH}$ [9]. Indeed more satisfactory results were obtained when the diazoketone and the catalyst were added simultaneously via two separate syringes to a solution of $\mathrm{CH}_{2} \mathrm{Cl}_{2}$ and $\mathrm{MeOH}$ or by slow addition of diazoketone and $\mathrm{MeOH}$ to a solution of catalyst in $\mathrm{CH}_{2} \mathrm{Cl}_{2}$. The bis(oxazoline)copper complex II used for the decomposition of diazoketone 2 was prepared according to [6] and activated with phenylhydrazine in dichloroethane. Slow addition ( $24 \mathrm{~h}$ ) of 2 in dichloroethane to this solution afforded the expected product 7 .

The $\mathrm{Rh}_{2}(\mathrm{OAc})_{4}$-catalyzed reactions produce racemic mixtures of esters. For determination of the enantiomeric com- position, the intermediate ketenes were intercepted with (-)-(S)-citronellol, and separation of the diastereoisomeric esters was attempted by capillary GC. This proved to be impossible, however. On the other hand, interception of the ketenes with (-)-menthol failed. Finally, the enantiomeric composition of the products was achieved by reducing the methyl esters to alcohols with $\mathrm{LiAlH}_{4}$ and esterification of the latter with (+)-(S)- $\alpha$-methoxy- $\alpha$-phenyl- $\alpha$-(trifluoromethyl)acetyl chloride [10][11] to provide a mixture of diastereoisomeric esters (Scheme 2).

All of these diastereoisomeric esters are separable either by GC using a methylsilicone capillary column, or by ${ }^{1} \mathrm{H}-\mathrm{NMR}$ and ${ }^{19}$ F-NMR using a shift reagent $\left(\mathrm{Eu}(\mathrm{fod})_{3}\right)$.

As Table 2 shows, even under improved conditions the yields of the $\mathrm{Rh}_{2}(\mathrm{MEPY})_{4}$-catalyzed reactions are usually significantly below that of those catalyzed with $\mathrm{Rh}_{2}(\mathrm{OAc})_{4}$. Unfortunately, the enantiomeric excess in all of the reaction is very low, and never exceeds $21 \%$. In the one case where the chiral $\mathrm{Cu}^{\mathrm{I}}$ complex II was used, the e.e. is even below that obtained with $\mathrm{Rh}_{2}(\mathrm{MEPY})_{4}$. This result is surprising considering the high (up to $94 \%$ ) e.e.'s achieved with the $\mathrm{Rh}_{2}(\mathrm{MEPY})_{4}$ cat- alyst in the intramolecular cyclopropanation of allylic diazo esters [3]. There are two important differences between the allylic and the $\beta, \gamma$-unsaturated diazo esters. The first one concerns the presence of the $\mathrm{O}$-atom in the chain of the former, which might contribute to the efficiency of the reaction. It is known from $\mathrm{Rh}^{11}$ catalyzed intermolecularcyclopropenation [12] and intramolecular $\mathrm{C}, \mathrm{H}$-insertion reactions [13] that substrates containing oxygen give often better results than their hydrocarbon analogues. The second difference lies in the chain length connecting the carbenic centre to the reacting double bond. The intermediate leading to the vinylogous Wolff rearrangement is a bicyclo [2.1.0]pentan-2-one, which is much more strained than the homologous bicyclo [3.1.0]hexan-2-one, a perfectly stable compound (Scheme 3). The strain of these species must be in part reflected in the respective transition states. According to the Hammond postulate, the more strained transition state will occur later on the reaction coordinate. With respect to geometry, this means, that the metal-carbene distance will be longer, and interactions between the chiral ligands of the metal and the substituents of the carbene decrease, which results in loss of stereochemical

Table 2. Yield and Enantiomeric Excess in $R h_{2}(M E P Y)_{4}$-Catalyzed Vinylogous Rearrangements

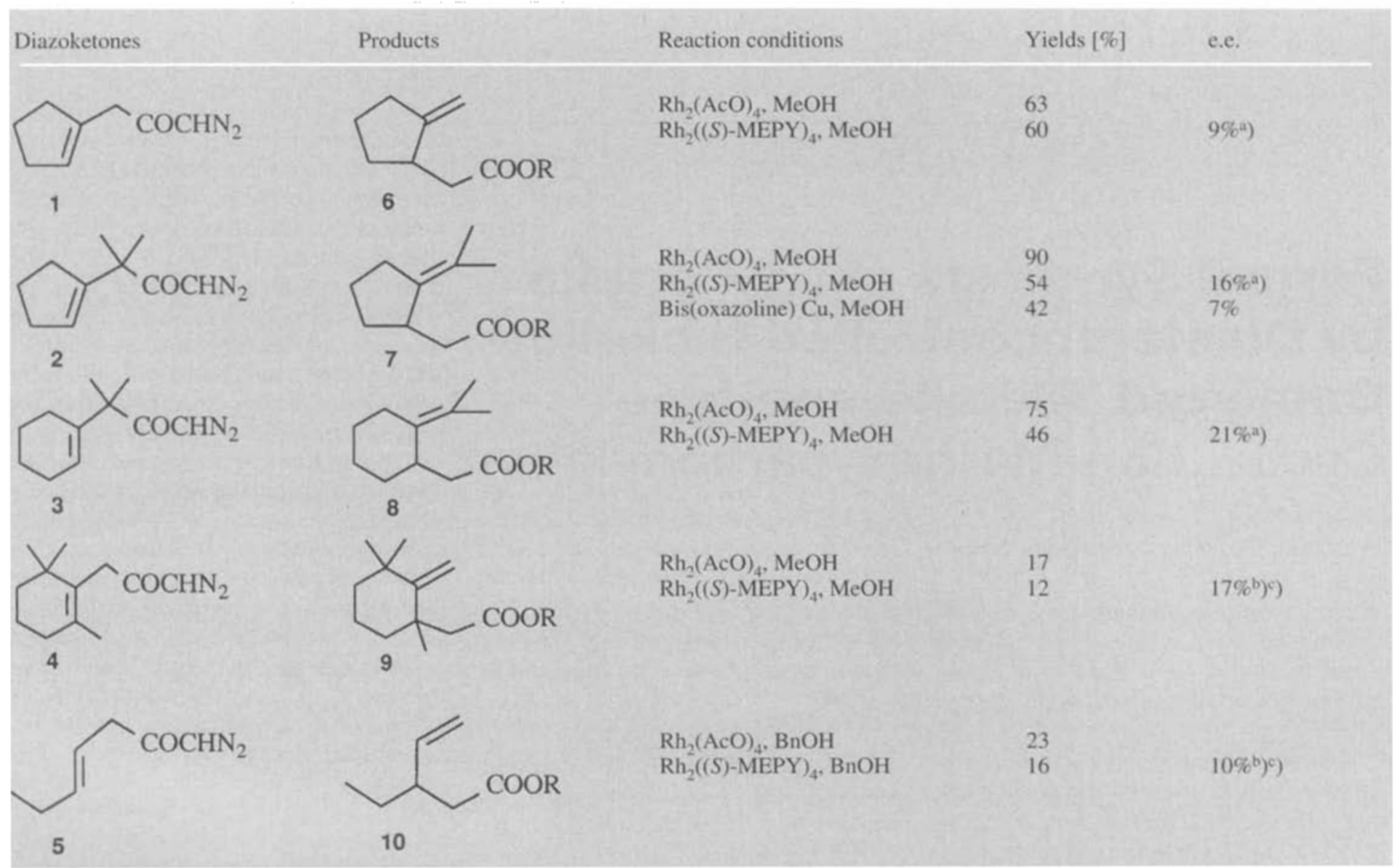

a) Determined by GC using a methylsilicone capillary column. ${ }^{\text {b) }}$ Determined by ${ }^{1} \mathrm{H}-\mathrm{NMR}$. ${ }^{\mathrm{c}}$ ) Determined by ${ }^{19} \mathrm{~F}-\mathrm{NMR}$ using a shift reagent (Eu(fod) $)_{3}$ ). 
Scheme 3
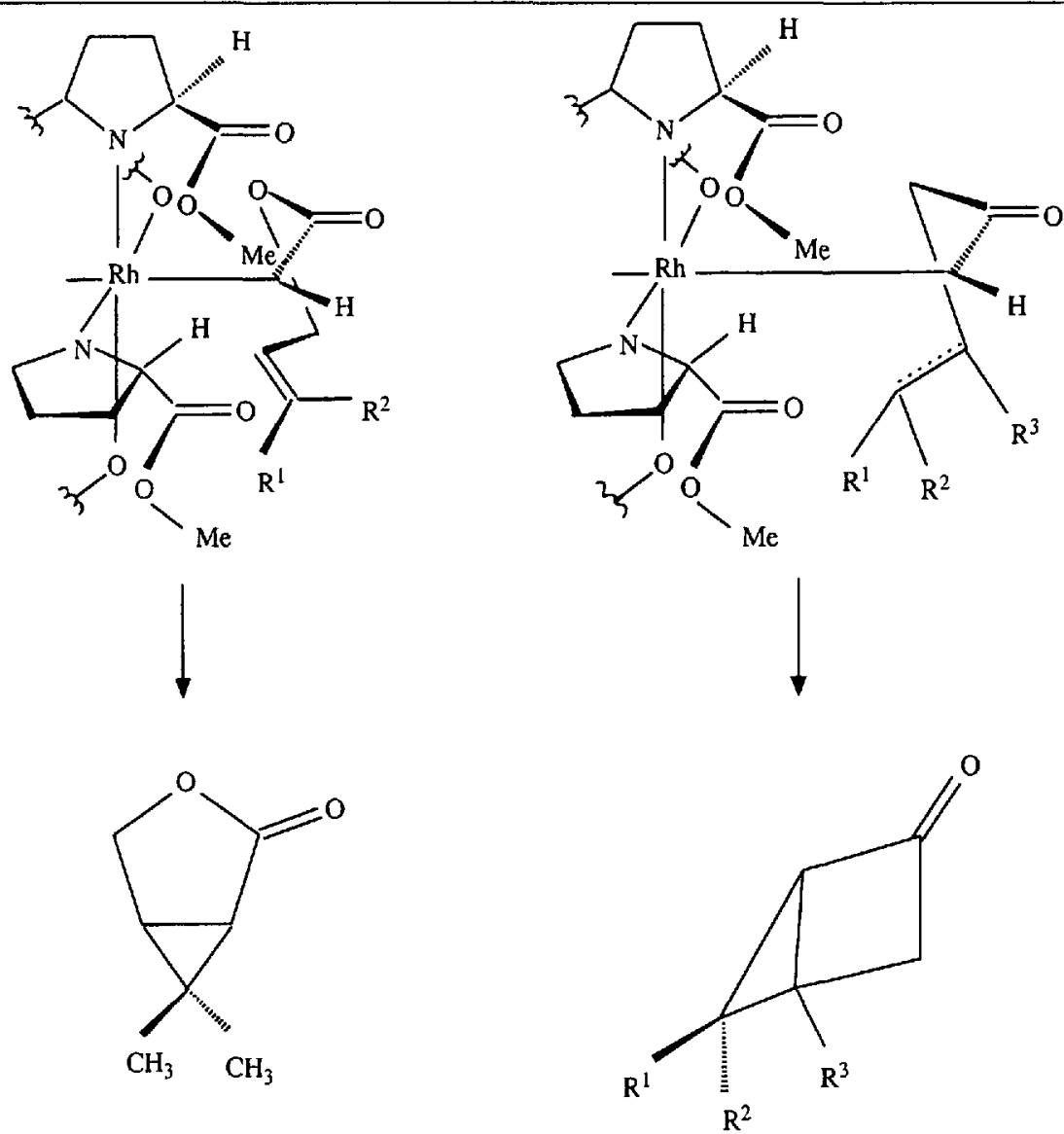

control. At the same time, the distance between the carbene and the double bond decreases, and one might expect more influence by the olefinic substituents. This is, however, compensated, because the substituents move away from the carbenic centre owing to rehybridization of the olefinic C-atoms.
This work was supported by the Swiss $\mathrm{Na}$ tional Science Foundation (project No. 20-27. 466.89). The authors are indebted to Prof. $A$. Pfaltz for helpful hints for the preparation of II.

Received: January 9, 1992

[1] A.B. Smith, B.H. Toder, S.J. Branca, J.Am. Chem. Soc. 1984, 106, 3995.

[2] A.B. Smith, B.H. Toder, R.E. Richmond, S.J. Branca, J. Am. Chem. Soc. 1984, 106, 4009.

[3] M.P. Doyle, R.J. Pieters, S.F. Matin, R.E. Austin, C.J. Oalmann, P. Müller, J. Am. Chem. Soc. 1991, 113, 1423.

[4] D. Müller, G. Umbricht, B. Weber, A. Pfaltz, Helv. Chim. Acta 1991, 74, 232.

[5] D.A. Evans, K.A. Woerpel, M.M. Hinman, M.M. Faul, J. Am. Chem. Soc. 1991, II3, 726.

[6] R.E. Lowenthal, A. Abiko, S. Masamune, Tetrahedron Lett. 1990, 3I, 6005.

[7] J.P. Lockensgard, J. O'Dea, E.A. Hill, J. Org. Chem. 1974, 39, 3355.

[8] S.J. Branca, R.L. Lock, A.B. Smith, J. Org. Chem. 1977, 42, 3165 .

[9] M.P. Doyle, private communication.

[10] J.A. Dale, D.L. Hull, H.S. Mosher, J. Org. Chem. 1969, 34, 2543.

[11] J.A. Dale, H.S. Mosher, J. Am. Chem. Soc. 1973, 95, 512.

[12] M.N. Protopova, M.P. Doyle, D. Ene, P. Müller, J. Am. Chem. Soc. 1992, accepted for publication.

[13] M.P. Doyle, A.van Oeveren, L.J. Westrum, M.N. Protopova, T.W. Clayton, J. Am. Chem. Soc. 1991, 113, 8982.

actone E methyl ester [3], (+)-3-isorauniticine [4], and, most recently, [5.5.5.5]fenestranes [5]. We present here a rational application of this process in a synthesis of

\section{Formal Synthesis of $( \pm)$-Coriolin} by Diastereocontrolled Nickel(0)Catalyzed 'Metallo-ene-type' Cyclization/Methoxycarbonylation

\section{Wolfgang Oppolzer* and Akira Ando}

Abstract. Bicyclooctanone $( \pm)-2$, an advanced intermediate for the synthesis of $( \pm)$ coriolin, has been synthesized in ten steps starting from 2,2-dimethylpent-4-enal (7). The key step $6 \rightarrow 3+11$ is a highly diastereoselective, $\mathrm{Ni}^{0}$-catalyzed, tandem intramolecular alkene allylation/carbonylation reaction.

The development and creative application of transition-metal-catalyzed reactions is presently at the forefront of organic synthesis [1]. Thus, recently discovered $\mathrm{Pd}^{0}$ - and $\mathrm{Ni}^{0}$-catalyzed intramolecular alkene (alkyne) allylations I $\rightarrow$ II show attractive perspectives for the stereocontrolled construction of various carbo- and heterocyclic systems (Scheme 1) [2].

This holds particularly for the tandem allylation/carbonylation I $\rightarrow$ II $\rightarrow$ III as illustrated by the syntheses of pentalenolthe triquinane terpenoid coriolin which features the topological bias of a preexisting stereocenter over developing stereocenters in the carbometalation step $\mathbf{I} \rightarrow$ II. Coriolin, a metabolite from the Basidiomycete Coriolus consors has been assigned structure 1 (Scheme 2) [6].

Reports of antibiotic and antitumor activity contributed to the popularity of 1 as a test case for cyclopentenone-annulation methodology [7]. The synthesis of ( \pm -1, reported by Exon and Magnus thus proceeds via the bicyclo[3.3.0]octanone $\mathbf{2}$, in turn assembled by means of a stereoselective intramolecular Pauson-Khand process [7e].

*Correspondence: Prof. W. Oppolzer Département de Chimie Organique Université de Genève $\mathrm{CH}-1211$ Genève 4 